\title{
EDITORIAL
}

\section{Korean Global Data Assimilation and Prediction System}

\author{
Song-You Hong ${ }^{1}$, Seon Ki Park ${ }^{2}$, Rokjin Park ${ }^{3}$, and Jimy Dudhia ${ }^{4}$ \\ ${ }^{1}$ Korea Institute of Atmospheric Prediction Systems (KIAPS), Seoul, Korea \\ ${ }^{2}$ Department of Environmental Science and Engineering, Ewha Womans University, Seoul, Korea \\ ${ }^{3}$ School of Earth Environment and Environmental Sciences, Seoul National University, Seoul, Korea \\ ${ }^{4}$ Mesoscale/Microscale Meteorology Laboratory (MMM), National Center for Atmospheric Research (NCAR), Boulder, Colorado, USA
}

(C) The Korean Meteorological Society and Springer 2018

The Korea Meteorological Administration (KMA) first started global Numerical Weather Prediction (NWP) in the late 1990's, for which the global data assimilation and prediction system had been adopted from the Japan Meteorological Agency (JMA). Having some difficulties in sustaining forecast skill, which was probably due to a system software issue in porting across computer platforms during the first several years of the 2000s, the KMA decided to adopt the United Kingdom Met Office's Unified Model (UM), and this has been operational since 2011 (Park et al., 2017). The UM-based numerical weather prediction system is a major component for providing daily weather forecasts in Korea. At the same time, the KMA launched a mission to develop major components of the model autonomously and established the Korea Institute of Atmospheric Prediction Systems (KIAPS) in 2011. Educative experience at KMA from using foreign NWP systems has shown limitations in improving the forecast performance, especially incorporating changes to allow for suggestions and evaluations by the KMA forecasters; thus, necessitating the development of KMA's own NWP system.

This special issue highlights the fully-fledged efforts in the development of the new Korean global data assimilation and prediction system. As of February 2018, the 12-km Korean Integrated Model (KIM) system has been launched in a real-time forecast framework, with a spectral-element non-hydrostatic dynamical core on a cubed sphere grid and a state-of-the-art physics parameterization package. The initial conditions are obtained via an advanced method of hybrid four-dimensional ensemble variational data assimilation (4DEnVar) over its native grid.

In their 2017 report, the Science Advisory Committee (SAC)* stated that they believe KIAPS addresses with great professionalism and creativity all the important aspects of NWP: dynamical core, coupled systems, physics, and data assimilation, at all time and space scales relevant to regional and global atmospheric forecasts. The SAC continues to encourage the team to collaborate and communicate with other major operational centers and to release KIM in the public domain in the long run, especially to share physics schemes or other software allowing for open discussion in scientific and technical publications. Through this

Corresponding Author: Song-You Hong, Korea Institute of Atmospheric Prediction Systems (KIAPS) 4F, 35 Boramae-ro 5gil, Dongjak-gu, Seoul 07071, Korea.

E-mail: songyou.hong@kiaps.org sharing, KIAPS can leverage its limited resources to benefit from contributions by international scientists with similar interests. In this manner, the KIAPS team aim eventually to attain the full maturity of a NWP R\&D group having a high international reputation and to achieve recognition on their own merits.

This issue is composed of eleven papers written by the KIAPS staff. S. Hong et al. overviews the development strategy and ongoing efforts of KIM, along with the evolution of its performance. S. Choi analyzes the structure of eigenvalues of the KIM dynamic core to clarify the issue of numerical stability. J. Kang et al. and I. Kwon et al. summarize the data processing and quality control, and the benefits of 4DEnVar, respectively. H. Song et al. and S. Shin et al. document the advanced technologies required for 4DEnVar over the cubed-sphere grid. Hong and Jang describe the impact of shallow convection processes on a simulated climatology. E. Lee et al. document the turbulent mixing in stratocumulus-topped boundary layer of NWP models. H. Choi et al. document the effects of spectral non-orographic gravity wave drag on weather and climate modeling. J. Kim et al. and K. Kim et al. present the scalability and computational efficiency issues of KIM.

We hope that the papers in this issue will stimulate further development of the KIM NWP system, and promote confidence in its use as a tool for further improving our scientific knowledge of weather and climate.

The KIAPS activity has been reviewed annually by SAC. Martin Miller served the chair of SAC from 2011 to 2015, followed by Gilbert Brunet until 2017. As of May 2018, Dale Barker serves the chair of the committee with Eugenia Kalnay, Joe Klemp, Richard Forbes, Wei-Kuo Tao, Stan Benjamin, Vijay Tallapragada, and Stephane Belair.

Acknowledgments. We, the guest editors of the Asia-Pacific Journal of Atmospheric Sciences (APJAS), are grateful to all the authors, reviewers, and the Korean Meteorological Society (KMS) and APJAS editorial staff.

\section{References}

Park, S., D.-J. Kim, S.-W. Lee, K.-W. Lee, J. Kim, E.-J. Song, and K.-H. Seo, 2017: Comparison of extended medium-range forecast skill between KMA ensemble, ocean coupled ensemble, and GloSea5. Asia-Pac. J. Atmos. Sci., 53, 393-401. 